\title{
Déficits Primários e Secundários de Funções Executivas Pós-TCE: análise de dissociações
}

\author{
Renata Kochhann \\ Natalie Pereira \\ Laura Damiani Branco \\ Charles Cotrena \\ Caroline de Oliveira Cardoso \\ Maila Rossato Holz \\ Nicolle Zimmermann \\ Rochele Paz Fonseca* \\ Pontifícia Universidade Católica do Rio Grande do Sul, Porto Alegre, Rio Grande do Sul, Brasil
}

\begin{abstract}
RESUMO
Este estudo avaliou as associações e dissociações encontradas entre déficits de memória episódica em relação aos de funções executivas e verificou se estes déficits encontrados eram primários ou secundários. Os 81 pacientes pós-Traumatismo Cranioencefálico (TCE) foram avaliados por meio do Teste de Aprendizagem Auditivo-Verbal de Rey e do Teste Hayling. Os resultados sugerem que prejuízo na velocidade de controle inibitório pode contribuir para déficit na memória episódica e que as velocidades de iniciação e inibição parecem ser complementares, mas a primeira precede a segunda. Nossos achados ressaltam que os prejuízos executivos provavelmente sejam primários em nossa amostra de pacientes pós-TCE e que estes prejuízos podem causar déficits na memória episódica.
\end{abstract}

Palavras-chave: traumatismo cranioencefálico; memória episódica; funções executivas; iniciação; inibição.

\section{ABSTRACT \\ Primary and Secondary Executive Function Deficits Following TBI: analysis of dissociations}

The present study evaluated the presence of associations and dissociations between impairments in episodic memory and executive functions in patients with traumatic brain injury (TBI), and verified whether these deficits were primary or secondary. Eighty-one patients with TBI were assessed using the Rey Auditory Verbal Learning Test and the Hayling Test. The results suggest that impairments in inhibition speed may contribute to deficits in episodic memory, and that initiation and inhibition abilities may be complementary and the first precedes the second. Our findings highlighted that primary executive impairment following TBI may lead to episodic memory deficits.

Keywords: traumatic brain injury; episodic memory; executive functions; initiation; inhibition.

Dentre os processos cognitivos mais investigados nas últimas décadas na literatura neuropsicológica, destacam-se as funções executivas (FEs) (Calhoun, 2006; Fonseca et al., 2011). Este termo refere-se a processos cognitivos orientados a objetivos, como resolução de problemas, planejamento, resistência à distração, flexibilidade cognitiva, habilidade de lidar com estímulos em situações desconhecidas e processo de inibição (Anderson \& Knight, 2010; Chan, Shum, Toulopoulou \& Chen, 2008).

\footnotetext{
Endereço para correspondência: Rochele Paz Fonseca - rochele.fonseca@gmail.com
} 
Dada a importância das FEs para a funcionalidade e qualidade de vida, assim como a alta incidência de prejuízos nestas habilidades após quadros neurológicos, como o Traumatismo Cranioencefálico (TCE) (Skand-sen et al., 2010; Hillary et al., 2010; Wood \& Liossi, 2007), a relação com as demais funções cognitivas tem sido detalhadamente investigada. Diversos estudos têm sido desenvolvidos com o objetivo de propor modelos teóricos para as FEs, assim como de especificar a relação entre tais habilidades e processos mnemônicos, atencionais e linguísticos (Busch, McBride, Curtiss, \& Vanderploeg, 2005; Diamond, 2013; Noël et al., 2012; Packwood et al., 2011).

Dentre os primeiros estudos que objetivaram desenvolver um modelo teórico abrangente e preciso para a compreensão das FEs, destaca-se o modelo proposto por Miyake e colaboradores (2000), no qual análises fatoriais foram utilizadas para identificar três componentes-chave das FEs: shifting, ou a alternância entre tarefas ou estratégias; updating, ou a atualização de conteúdo na memória de trabalho e outros processos cognitivos que necessitam de constante monitoramento e modificação de informações on-line; e inibição, que se refere à capacidade de interromper comportamentos automáticos e, frequentemente, substituí-los por outros menos automatizados, porém mais adaptativos a um contexto específico (Miyake et al., 2000).

Mais recentemente, uma abordagem diferenciada foi utilizada por Packwood, Hodgetts e Tremblay
(2011) para investigar as FEs e desenvolver um modelo teórico capaz de detalhar o funcionamento de diferentes processos executivos. As cinco funções principais encontradas por Packwood et al. (2011) consistiram no planejamento, memória de trabalho, shifting, inibição e fluência. Por meio do estudo da relação entre componentes executivos e seus subcomponentes, além de outras habilidades cognitivas semântica e hierarquicamente associadas a eles, Packwood et al. (2011) diferenciaram as FEs de habilidades mnemônicas, atencionais e linguísticas, de modo que foi possível identificar as distinções entre estas habilidades, assim como sua interdependência, e compreender a razão da existência de dissociações entre elas em pacientes com diferentes condições clínicas. Já Diamond (2013) também buscou desenvolver um modelo explicativo das FEs a partir de uma revisão de estudos empíricos e teóricos anteriormente divulgados na literatura. A autora identificou quatro componentes principais das FEs: a memória de trabalho, a flexibilidade cognitiva, o controle inibitório e funções superiores, como a resolução de problemas, o raciocínio e o planejamento. Este modelo associa as FEs com diversas outras habilidades cognitivas, como, por exemplo, as habilidades atencionais, associadas às FEs pelo papel do componente inibitório na atenção seletiva ou concentrada (Figura 1). O componente inibitório também seria responsável pela relação entre FEs e o comportamento social. Já a inteligência fluida teria relação com as FEs por meio dos seus componentes mais complexos como o planejamento e o raciocínio.

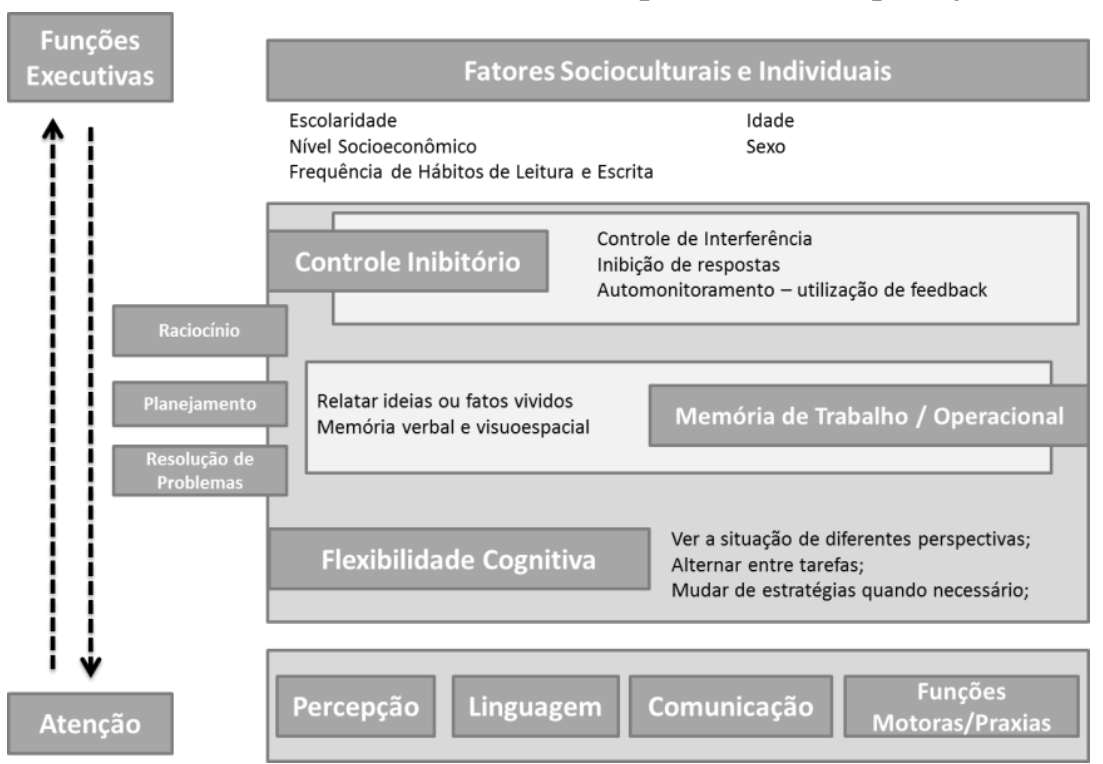

Figura 1. Adaptação do modelo sugerido por Diamond, 2013. 
Observa-se, dessa forma, uma complexidade crescente dos modelos de FEs desenvolvidos na literatura, assim como tentativas progressivamente mais elaboradas de integrar as FEs a outras habilidades cognitivas. Um processo cognitivo pouco estudado é a memória episódica e sua relação com as funções executivas, diferentemente da memória de trabalho, que é considerada uma função executiva (Packwood et al., 2011; Diamond, 2013). A memória episódica é um conceito fluido (Baddeley et al., 2011; Tulving, 2002), que difere dos outros sistemas de memória por se utilizar do contexto, tanto de tempo quanto de espaço, em que as informações foram adquiridas, no momento de sua evocação. Caracteriza-se por não ser apenas a evocação de uma lembrança retida (Lezak, Howieson \& Loring, 2004), mas um sistema integrado que registra informações de episódios vivenciados para serem recuperadas quando necessário e evocadas em determinado momento, podendo ser acessada a partir de uma re-experiência consciente dos eventos passados (Strauss, Sherman, \& Spreen, 2006).

Nesse sentido, queixas relacionadas à memória episódica encontradas no contexto clínico referem-se à aprendizagem limitada, ao rápido esquecimento de informações aprendidas, e à dificuldade de reconhecimento (Greenaway, et al., 2006). Essas queixas relacionadas ao prejuízo da memória episódica podem estar presentes em quadros pós-TCE. Como as características clínicas e neurológicas do TCE são heterogêneas e, em alguns casos, as lesões no tecido cerebral não são evidentes no momento do traumatismo, a investigação clínica é importante para que sejam investigadas lesões secundárias e descartados outros sintomas (Maas, Stocchetti, \& Bullock, 2008).

Ressalta-se a necessidade de estudar os déficits ocasionados por essa patologia, já que o TCE é considerado um importante problema de saúde pública, de maior incidência em indivíduos adultos jovens, e considerado a maior causa de morte e déficits/sequelas em todo o mundo (Selassie et al., 2008; Roozenbeek, Maas, \& Menon, 2013). No Brasil, embora tenham ocorrido diversas campanhas e políticas públicas alertando sobre os riscos do excesso de velocidade e do abuso de álcool associados ao trânsito, atualmente uma em cada seis internações nos prontos-socorros é devida ao TCE, sendo a maior parte delas associadas ao trânsito (Batista, Baccani, Silva, Gualda, \& Vianna Jr, 2005; Maia et al., 2013).
Prejuízos nas FEs pós-TCE são bem descritos em estudos com amostras de TCE de leves (Lipton et al., 2009) a graves (Kavé et al., 2011). Conexões axonais podem ser afetadas em diferentes níveis, dependendo da gravidade do TCE. No entanto, foi detectado em estudos com imagem por tensor de difusão que há disfunção axonal até mesmo no TCE leve (Lipton et al., 2009; Zappala, de Schotten \& Eslinger, 2012). Lesões focais, como hematoma, hemorragia e contusões também são frequentes e podem afetar diferentes estruturas cerebrais, e os estudos relatam uma alta incidência de danos em lobos frontais e temporais (Bigler, 2003), regiões cerebrais que estão associadas ao funcionamento executivo e mnemônico. Os déficits nas FEs têm sido demonstrados em componentes tais como a inibição, fluência verbal (Skandsen et al., 2010), velocidade de processamento da informação (Hillary et al., 2010) e de flexibilidade cognitiva (Wood \& Liossi, 2006).

Adicionalmente, um estudo realizado com pacientes após 10 anos do TCE e controles pareados demonstrou que o grupo de lesão apresentou um desempenho significativamente pior nas tarefas do RAVLT, Hayling e SART. A presença de um pior desempenho refere que a amostra consiste com uma alta frequência de lesões frontais e que essas deficiências cognitivas que afetam o domínio da velocidade de processamento, memória episódica e funcionamento executivo estão presentes após 10 anos do TCE e estão associadas à gravidade da lesão (Draper, \& Ponsford, 2008). Os dados deste estudo demonstram a necessidade de ser realizada uma avaliação neuropsicológica em pacientes que sofreram TCE, mesmo que ela seja realizada vários anos após a lesão, pois prejuízos cognitivos ainda poderão ser encontrados nos pacientes e estar prejudicando sua qualidade de vida.

Por isso, o estudo da relação entre as habilidades cognitivas mostra-se necessário a fim de auxiliar na compreensão de sequelas provenientes de quadros neurológicos que afetam a funcionalidade do indivíduo e nos estudos neuropsicológicos a respeito de associações e dissociações entre déficits cognitivos em pacientes acometidos por diferentes patologias. Nesse contexto, a associação caracteriza-se pelo prejuízo em duas tarefas ou dois componentes cognitivos por influência de uma mesma variável, indicando que talvez processamentos em comum estejam na base (Fonseca et al., 2012). Em contrapartida, o conceito de 
dissociação simples consiste em diferentes padrões de desempenho (com/sem prejuízo) entre duas tarefas quando se avalia um mesmo caso, ou seja, quando um indivíduo apresenta déficit em um componente cognitivo acompanhado de preservação de outro componente cognitivo. Em vista de complementar tal quadro, as dissociações duplas investigam padrões contrários de desempenho entre dois casos em duas tarefas, ou seja, enquanto um paciente demonstra desempenho intacto em uma tarefa e prejudicado em outra, um segundo paciente apresenta o padrão inverso (Davies, 2010; Fonseca et al., 2012). Outra maneira de aprimorar a avaliação dos déficits pode ser com a magnitude do prejuízo encontrado, pois quanto maior for o déficit maiores as probabilidades de que seja primário. Consideram-se déficits primários aqueles atribuídos exclusivamente a um prejuízo na função identificada, enquanto os secundários seriam os que se encontram prejudicados devido ao prejuízo em outra função cognitiva (Borosh \& Johnson, 2007).

Portanto, o estudo da relação entre habilidades cognitivas mostra-se essencial para a compreensão de sequelas provenientes de quadros neurológicos, como em casos pós-TCE. Embora as FEs tenham sido muito estudadas pós-TCE, ainda é incipiente a investigação da relação entre subcomponentes executivos e mnemônicos episódicos assim como a relação entre subcomponentes executivos, tais como iniciação e inibição, por exemplo. Nesse sentido, somente foi encontrado um estudo que integrou o funcionamento da memória episódica com componentes das FEs em pacientes pós-TCE (Busch et al., 2005). Assim, o objetivo deste estudo foi avaliar as associações e dissociações encontradas entre déficits de um teste que avalia memória episódica em relação a outro que avalia FEs em um grupo de pacientes pós-TCE e verificar se os déficits poderiam ser considerados primários ou secundários a partir dos escores $\mathrm{Z}$ encontrados.

\section{MÉTODO}

\section{Amostra}

Participaram do presente estudo 81 indivíduos ( $\mathrm{n}=$ 22 do sexo feminino e $\mathrm{n}=59$ do sexo masculino) pósTCE recrutados de hospitais de Porto Alegre, Rio Grande do Sul, Brasil. Os participantes, com idades entre 18 e 72 anos $(M=36,80 ; D P=14,67)$, tinham um mínimo de 2 e um máximo de 19 anos de escolaridade formal sem a inclusão de repetências $(\mathrm{M}=$ 9,72; DP = 3,74). Trinta e dois participantes apresentavam TCE grave, enquanto um indivíduo apresentava TCE moderado, e 48, TCE leve.

A amostra foi recrutada com base nos seguintes critérios de inclusão: no mínimo 18 anos de idade; diagnóstico de TCE leve, moderado ou grave por meio de escores na Glasgow Coma Scale (Teasdale, \& Jennett, 1974) no momento da hospitalização (coletado de registros hospitalares); autorrelato da duração da perda de consciência após o trauma (menos de 30 minutos - leve; 30 minutos a 24 horas - moderado; mais de 24 horas - grave) ou duração da amnésia póstraumática (menos de 24 horas - leve; um a sete dias moderado; mais de sete dias - grave) (Iverson, \& Lange, 2011); ausência de histórico de quadros neurológicos além do TCE; TCE fechado. Participantes analfabetos ou que apresentassem dificuldades para realizar as tarefas neuropsicológicas propostas (p. ex. pacientes sedados, acamados, ou sofrendo dores intensas) foram excluídos do estudo.

\section{Instrumentos e procedimentos}

Os participantes foram avaliados sob condições adequadas para a condução de tarefas neuropsicológicas, em locais silenciosos e ventilados, após ler e assinar um termo de consentimento livre e esclarecido. Este estudo foi aprovado pelo CEP da PUCRS (10/05134). Participantes foram avaliados com as seguintes tarefas:

- Teste Hayling - (Burguess \& Shallice, 1996; adaptado para o português brasileiro por Fonseca Oliveira, Gindri, Zimmermann, \& Reppold, 2010). As funções avaliadas por este instrumento são a velocidade de processamento, iniciação, processo de inibição e flexibilidade cognitiva. O teste é estruturado em duas partes (A e B), cada uma contendo 15 frases nas quais está omitida a última palavra. As frases são lidas em voz alta para o paciente, que deve completá-las o mais rápido possível com uma palavra que dê sentido a frase proferida (parte A) ou que não tenha qualquer ligação lógica com a frase (parte B). As variáveis mensuradas são o tempo de latência até a produção da resposta, o número de acertos e o número de erros. Ainda, é calculada a diferença entre o tempo necessá- 
rio para completar as duas partes do teste a fim de avaliar a velocidade de estratégias inibitórias utilizadas pelo paciente. Por último, na parte $\mathrm{B}$ do teste, é calculado um escore qualitativo com base na natureza dos erros cometidos pelo paciente, em que escores mais altos indicam pior controle inibitório.

- Teste de Aprendizagem Auditivo-Verbal de Rey (RAVLT) (Rey, 1958; adaptado para o português brasileiro por Malloy-Diniz et al., 2000). O instrumento mede componentes da memória episódica verbal, como a memória de curto e longo prazo, memória de reconhecimento, aprendizagem verbal e suscetibilidade à interferência. $\mathrm{O}$ teste contém 15 substantivos (lista A), que são lidos para o paciente cinco vezes consecutivas, cada uma seguida por um teste de lembrança. Depois, uma lista de interferência (lista B) é apresentada, seguida por um teste de lembrança. Posteriormente pede-se ao paciente que recorde as palavras da lista A (A6), sem reapresentá-las. Após 20 minutos, testa-se mais uma vez a lembrança da lista A (A7). Finalmente, testa-se o reconhecimento por meio de uma lista de 50 palavras. As variáveis mensuráveis são Learning Over Trials (LOT) (soma total de palavras recordadas de A1 até A5 - (5 x A1), que permite verificar a curva de aprendizagem de palavras durante A1 a A5, as taxas de interferência proativa (ITP) (B1/A1) e a interferência retroativa (ITR) (A6 / A5).

\section{Análise de dados}

Análises inferenciais e descritivas foram realizadas utilizando o software Statistical Package for the Social Sciences (SPSS), versão 17.0. Os escores brutos obtidos por pacientes em cada tarefa foram convertidos em escores $\mathrm{Z}$ com base em médias e desviospadrão (DP) obtidos para amostras comparáveis de pacientes saudáveis. $\mathrm{O}$ desempenho foi considerado prejudicado quando escores $\mathrm{Z}$ eram iguais ou inferiores a -1,5 DP, de acordo com o ponto de corte estabelecido por Kavé et al. (2011) e Schoenberg et al. (2006). A comparação da distribuição entre grupos com e sem déficits por componentes/escores - análise de associações e dissociações entre o desempenho de pacientes em cada tarefa - foi efetuada pelo Teste Qui-quadrado.

\section{RESULTADOS}

$\mathrm{Na}$ amostra total, somente houve diferença significativa entre as associações e dissociações dos déficits no tempo B do Teste Hayling (componente de velocidade de inibição) e no learning over time (LOT) do RAVLT (componente de aprendizagem) $(\mathrm{p}=0,020)$ (Tabela 1), e entre os déficits no tempo A do Teste Hayling (componente de velocidade de iniciação) e no tempo B do Teste Hayling (componente de velocidade de inibição) (Tabela 2).

Tabela 1

Frequência de Déficits no Tempo B do Teste Hayling e no Learning Over Time (LOT) do RAVLT

\begin{tabular}{llll} 
& & RAVLT LOT & \\
& & Adequado & Deficitário \\
& & Observado/esperado & Observado/esperado \\
\cline { 3 - 3 } & Adequado & $34 / 29$ & $14 / 19$ \\
Hayling Tempo B & & $69,40 \%$ & $43,80 \%$ \\
& Deficitário & $15 / 20$ & $18 / 13$ \\
& & $30,60 \%$ & $56,30 \%$ \\
& & $\mathbf{p}=\mathbf{0 , 0 2 0}$ \\
\hline
\end{tabular}


Tabela 2

Frequência de Déficits no Teste Hayling Tempo A e Teste Hayling Tempo B

\begin{tabular}{llll} 
& & Hayling Tempo B & \\
& & Adequado & Deficitário \\
& & Observado/esperado & Observado/esperado \\
\cline { 3 - 3 } & & $37 / 33$ & $18 / 22$ \\
Hayling Tempo A & Adequado & $67,3 \%$ & $32,7 \%$ \\
& & $11 / 15$ & $15 / 11$ \\
& Deficitário & $42,3 \%$ & $57,7 \%$ \\
& & & $\mathbf{p}=\mathbf{0 , 0 3 0}$ \\
\hline
\end{tabular}

Adicionalmente, as análises descritivas demonstraram que, dos 18 pacientes da amostra que apresentaram associação de déficits no funcionamento executivo e no funcionamento mnemônico, os déficits no componente executivo de velocidade de inibição $(\mathrm{M}=$ $-3,75$; $\mathrm{DP}=1,99$ ) foram maiores do que os déficits no componente de aprendizagem $(\mathrm{M}=-2,43$; $\mathrm{DP}=0,75)$. Em contrapartida, as análises descritivas demonstraram que dos 15 pacientes que apresentaram associação de déficits nos componentes de iniciação e inibição, os déficits no componente de velocidade de iniciação $(M=-10,80 ; \mathrm{DP}=20,77)$ foram maiores do que os déficits no componente de velocidade de inibição ( $\mathrm{M}=-4,93 ; \mathrm{DP}=2,52)$.

Não houve diferença estatisticamente significante entre as associações ou dissociações dos déficits encontrados no tempo A do Teste Hayling (componente de velocidade de iniciação), ou no tempo B-A do Teste Hayling (componente de velocidade de estratégias inibitórias), ou nos erros do tempo B do Teste Hayling (componente de controle inibitório) em relação aos déficits encontrados no componente de aprendizagem (LOT) do RAVLT. Também não houve diferença estatisticamente significante entre as associações ou dissociações dos déficits encontrados no componente de velocidade de iniciação ou de inibição do Teste Hayling, ou no componente de velocidade de estratégias inibitórias ou de controle inibitório do Teste Hayling em relação aos déficits encontrados nos componentes de interferência proativa (ITP) e de interferência retroativa (ITR) do RAVLT.

\section{DISCUSSÃO}

Este estudo teve o objetivo de avaliar as associações e dissociações encontradas entre os componentes de aprendizagem avaliados pelo Teste de Aprendizagem Auditivo-Verbal de Rey (RAVLT) em comparação aos componentes de funções executivas avaliados pelo Teste Hayling em um grupo de pacientes pós-TCE e verificar se os déficits encontrados a partir dos escores $\mathrm{Z}$ seriam considerados primários ou secundários.

$\mathrm{Na}$ análise de ocorrência de déficits, os achados demonstram que houve mais associações do que dissociações entre estes escores. Ou seja, havia prejuízo tanto em componente do funcionamento mnemônico quanto do funcionamento executivo. Desse modo, tais déficits parecem co-ocorrer, indicando que talvez processamentos em comum estejam na base destes componentes cognitivos (Fonseca et al., 2012)

Nos resultados encontrados por meio do escore Z, foi observado que a velocidade de controle inibitório provavelmente seria um prejuízo primário de disfunção executiva em relação ao aprendizado, que parece ser secundário, pois o escore $\mathrm{Z}$ do primeiro componente foi maior do que o do segundo, então o componente que apresenta prejuízo maior (velocidade de inibição) provavelmente esteja causando ou piorando o componente que apresenta prejuízo menor (aprendizado). Estes dados sugerem que o funcionamento executivo pode estar mediando os prejuízos mnemônicos. Outros estudos têm sugerido que o prejuízo em um domínio cognitivo pode mediar o prejuízo de outras funções cognitivas (Borosh \& Johnson, 2007; Henry, Rendell, Kliegel, \& Altgassen, 2007). O controle inibitório é responsável por inibir estímulos e respostas irrelevantes ao contexto. Diamond (2013) sugere que o controle inibitório auxilia a memória de trabalho, pois, para conseguir manter a mente focada e relacionar várias ideias ou fatos juntos, deve-se ser 
capaz de inibir distrações internas e externas. Os resultados do presente estudo demonstram que, se a capacidade de controle inibitório estiver deficitária, pode influenciar no prejuízo de aprendizagem episódica, já que os pacientes apresentaram maior prejuízo no controle inibitório, que estava associado ao prejuízo na aprendizagem.

Os achados deste estudo entre aprendizagem e velocidade de inibição sugerem que a velocidade de controle inibitório pode influenciar a aprendizagem. Ou seja, o funcionamento executivo parece estar subjacente à aprendizagem auditivo verbal, e a própria lista do RAVLT, ao longo da realização das tentativas, pode criar uma interferência nela mesma se não houver um controle inibitório adequado. Um estudo que investigou os subcomponentes de habilidades executivas em pacientes pós-TCE (Busch et al., 2005) sugeriu que a falha em inibir o relato das palavras em um teste de memória verbal deve estar relacionada com o papel do córtex frontal na evocação das memórias.

Achados similares foram demonstrados em alcoolistas sóbrios comparados a controles (Noël et al., 2012), quando os autores, por meio de uma análise de regressão, verificaram se o desempenho das funções executivas poderia explicar o fraco desempenho na memória episódica. Os resultados encontrados foram que os alcoolistas apresentam uma memória episódica verbal (California Verbal Learning Test) prejudicada nos processos de codificação e recuperação, e esses prejuízos foram também explicados, devido a déficits no raciocínio, inibição, coordenação de duas tarefas e flexibilidade cognitiva. Isso reforça que déficits no funcionamento executivo podem estar relacionados a distúrbios na memória episódica (Noël et al., 2012).

Adicionalmente, um estudo com pacientes com cirrose hepática mínima comparados a controles encontrou prejuízos no reconhecimento e na interferência retroativa, avaliados pelo RAVLT, e na velocidade de processamento da parte A e B do Trail Making Test. Estes resultados sugerem que houve uma disfunção na codificação e armazenamento da informação destes pacientes, além de déficits na recuperação da memória e um leve prejuízo na flexibilidade cognitiva (Torres, Abrantes \& Brandão-Mello, 2013). Alguns estudos referem que comprometimentos do sistema medial temporal e do córtex pré-frontal podem trazer prejuízos na memória episódica e na velocidade de proces- samento (Gagnon et al., 2010; Squire et al., 2004; Torres et al., 2013).

Outro achado significativo em nosso estudo foi encontrado entre os escores de velocidade de iniciação (Tempo A) e de inibição (Tempo B) do Hayling, pois $57,7 \%$ dos pacientes que apresentaram prejuízo na inibição também apresentaram prejuízo na iniciação. E 67,3\% dos pacientes que apresentaram desempenho adequado na inibição também apresentaram desempenho adequado na iniciação. Esta relação entre os componentes de velocidade de iniciação e inibição sugere ser complementar um em relação ao outro, pois necessitamos de ambos os processos para a realização da tarefa. Adicionalmente, os resultados encontrados com o escore $\mathrm{Z}$ também demonstram que a iniciação provavelmente é um prejuízo primário em relação à inibição, que parece ser secundário nesta amostra de pacientes pós-TCE. Achados similares foram previamente demonstrados a partir de uma alta incidência de danos em lobos frontais relatados em estudos com pacientes que sofreram TCE (Bigler, 2003) e relato de déficits nas FE foram demonstrados em componentes tais como a inibição (Skandsen et al., 2010), velocidade de processamento da informação (Hillary et al., 2010) e de flexibilidade cognitiva (Wood \& Liossi, 2006).

Embora as principais hipóteses explicativas para os achados encontrados seja a ocorrência de TCE, outros fatores de história de vida individuais, socioculturais que não foram mensurados neste estudo podem ter contribuído para isso. Adicionalmente, outra limitação deste estudo foi o uso de apenas um paradigma para memória episódica, sendo ele apenas a palavra. Sugerimos que próximos estudos utilizem o discurso como paradigma de avaliação de memória episódica e/ou mais de um paradigma, como o discurso associado ao uso de lista de palavras, para que prejuízos de memória em mais de um nível possam ser verificados.

Com o estudo da relação entre componentes executivos e seus subcomponentes, foi possível identificar as distinções entre estas habilidades, sua associação com processos mnemônicos episódicos em pacientes pós-TCE, bem como possíveis déficits primários e secundários. Ressalta-se a importância do uso dos testes RAVLT e Hayling na avaliação de prejuízo cognitivo em pacientes pós-TCE, pois conseguem identificar alterações funcionais mesmo após terem 
passado dez anos após o TCE (Draper \& Ponsford, 2008). Os resultados do presente estudo sugerem que a realização de análises intercomponentes entre diferentes testes pode aperfeiçoar os resultados para uma avaliação clínica, auxiliando na elaboração de diagnósticos neuropsicológicos.

\section{REFERÊNCIAS}

Anderson, T. M., \& Knight, R. G. (2010). The long-term effects of traumatic brain injury on the coordinative function of the central executive. Journal of Clinical and Experimental Neuropsychology, 32(10), 1074-1082. doi: 10.1080/13803391003733560.

Baddeley, A., Eysenck, M. W., \& Anderson M. C. (2011). Memória. Porto Alegre: Artmed.

Batista, S. E. A., Baccani, J. G., Silva, R. A. de P. e, Gualda, K. de P. F., \& Vianna Jr, R. J. de A. (2005). Análise comparativa entre os mecanismos de trauma, as lesões e o perfil de gravidade das vítimas, em Catanduva - SP. Revista do Colégio Brasileiro de Cirurgiões, 33(1), 610. doi:10.1590/S0100-69912006000100003.

Bigler, E. D. (2003). Neurobiology and neuropathology underlie the neuropsychological deficits associated with traumatic brain injury. Archives of Clinical Neuropsychology, 18, 595-621. doi: 10.1016/S0887-6177(02) 00156-7.

Borosh, B. \& Johnson, N. (2007). Neuropsychology of Frontotemporal Dementia and Primary Progressive Aphasia. Türk Nöroloji Dergisi, 13(1), 68-84. doi: 10.1177/089198 8708316856.

Burgess, P. W. \& Shallice, T. (1996). Response suppression, initiation, and strategy use following frontal lobe lesions. Neuropsychologia, 34, 1, 263-273. doi: 10.1016/ 0028-3932(95)00104-2.

Busch, R. M., McBride, A., Curtiss, G., \& Vanderploeg, R. D. (2005). The components of executive functioning in traumatic brain injury. Journal of Clinical and Experimental Neuropsychology, 27(8), 1022-1032. doi: $10.1080 / 13803390490919263$.

Calhoun, J. A. (2006). Executive Functions: A Discussion of the Issues Facing Children with Autism Spectrum Disorders and Related Disorders. Seminars in Speech and Language, 27(1), 60-72. doi: 10.1055/s-2006932439.

Chan, R. C. K., Shum, D., Toulopoulou, T., \& Chen, E. Y. H. (2008). Assessment of executive functions: Review of instruments and identification of critical issues. Archives of Clinical Neuropsychology, 23(2), 201-216. doi: 10.1016/j.acn.2007.08.010.

Davies, M. (2010). Double dissociation: Understanding its role in cognitive. Neuropsychology, 25(5), 500-540. doi: 10.1111/j.1468-0017.2010.01399.x.
Diamond, A. (2013) Executive Functions. Annual Review of Clinical Psychology, 64, 135-68. doi: 10.1146/ annurev-psych-113011-143750.

Draper, K., \& Ponsford, J. (2008). Cognitive Functioning Ten Years Following Traumatic Brain Injury and Rehabilitation. Neuropsychology, 22(5), 618-625. doi: 10.1037/0894-4105.22.5.618.

Fonseca, R. P., Oliveira, C., Gindri, G., Zimmermann, N., Reppold, C., \& Parente, M. A. M. P. (2010). Teste Hayling: Um instrumento de avaliação de componentes das funções executivas. In C. Hutz (Org.). Avaliação Psicológica e Neuropsicológica de crianças e adolescentes (1t ed., pp. 337-364). São Paulo: Casa do Psicólogo.

Fonseca, R. P., Pureza, J., Gonçalves, H., Grassi-Oliveira, R., Kristensen, C. H. \& Stein, L. M. (2011). Estudos sobre cognição humana na revista Psico nos últimos 40 anos. Psico, 42(3), 295-302.

Fonseca, R. P., Zimmermann, N., Pawlowski, J., Oliveira, C. R., Gindri, G., Scherer, L. C. \& Parente, M. A. M. P. (2012). Métodos em avaliação neuropsicológica. In J. Landeira-Fernandez \& S. S. Fukusima (Eds.), Métodos em avaliação neuropsicológica (1st ed., pp. 266-296). Barueri: Manole.

Gagnon, G., Blanchet, S., Grodin S. \& Scheider, C. (2010). Paired-pulse transcanial magnetic stimulation over the dorsolateral prefrontal cortex interferes with episodic encoding and retrieval for both verbal and non-verbal materials. Brain Research, 14 (44), 148-158. doi: 10.1016/ j.brainres.2010.04.041.

Greenaway, M. C., Lacritz, L. H., Binegar, D., Weiner, M. F., Lipton, A., \& Cullum, C. M. (2006). Patterns of verbal memory performance in mild cognitive impairment, Alzheimer Disease, and normal aging. Cognitive Behavioral Neurology, 19(2), 79-84. doi: 10.1097/01.wnn. 0000208290.57370.a3

Henry, J. D., Rendell, P. G., Kliegel, M. \& Altgassen, M. (2007). Prospective memory in schizophrenia: Primary or secondary impairment? Schizophrenia Research, 95, 179-185. doi: 10.1016/j.schres.2007.06.003.

Hillary, F. G., Genova, H. M., Medaglia, J. D., Fitzpatrick, N. M., Chiou, K. S., Wardecker, B. M., Franklin, R. G., \& De Luca, J. (2010). The nature of processing speed deficits in traumatic brain injury: Is less brain more? Brain Imaging and Behavior, 4(2), 141-54. doi: 10.1007/ s11682-010-9094-z.

Iverson, G. L., \& Lange, R. T. (2011). Moderate-Severe Traumatic Brain Injury. In M. R., Schoenberg and J. G. Scott (Eds.), The Black Book of Neuropsychology: A syndrome based approach, (pp. 663-689). New York: Springer.

Kavé, G., Heled, E., Vakil, E., \& Agranov, E. (2011). Which verbal fluency measure is most useful in demonstrating executive deficits after traumatic brain injury? Journal of Clinical and Experimental Neuropsychology, 33(3), 358-365. doi: 10.1080/13803395.2010.518703. 
Lezak, M. D., Howieson, D. B., \& Loring, D. W. (2004). Neuropsychological assessment. New York: Oxford University.

Lipton, M. L., Gulko, E., Zimmerman, M. E., Friedman, B. W., Kim, M., Gellella, E., Gold, T., Shifteh, K., Ardekani, B. A., Branch, C. A. (2009). Diffusion-tensor imaging implicates prefrontal axonal injury in executive function impairment following very mild traumatic brain injury. Radiology, 252(3), 816-824. doi: 10.1148/radiol. 2523081584.

Maas, A. I. R., Stocchetti, N., \& Bullock, R. (2008). Moderate and severe traumatic brain injury in adults. Lancet Neurology, 7(8), 728-741. doi: 10.1016/S1474-4422(08) 70164-9.

Maia, B., Paula, F., Cotta, G., Cota, M., Públio, P., Oliveira, H., \& Oliveira, T. (2013). Perfil Clínico-Epidemiológico das Ocorrências de Traumatismo Cranioencefálico. Revista Neurociências, 21(1), 43-52. doi: 10.4181/RNC.2013.21.786.10p.

Malloy-Diniz, L. F., da Cruz, M. F., Torres, V., \& Cosenza, R. (2000). O teste de aprendizagem auditivo-verbal de Rey: Normas para uma população brasileira. Revista Brasileira de Neurologia, 36(3), 79-83. doi: 10.1590/ S1516-44462006005000053.

Miyake, A., Friedman, N. P., Emerson, M. J., Witzki, A. H., Howerter, A. \& Wager, T. D. (2000). The unity and diversity of executive functions and their contributions to complex "Frontal Lobe" tasks: A latent variable analysis. Cognitive Psychology, 41(1), 49-100. doi: 10.1006/ cogp.1999. 0734.

Nöel, X., Van der Linden, M., Brevers, D., Campanella, S., Hanak, C., Kornreich, C. \& Verbanck, P. (2012). The contribution of executive functions deficits to impaired episodic memory in individuals with alcoholism. Psychiatry Research, 198, 116-122. doi: 10.1016/j.psychres. 2011.10.007.

Packwood, S., Hodgetts, H. M. \& Tremblay, S. (2011). A multiperspective approach to the conceptualization of executive functions. Journal of clinical and experimental neuropsychology, 33(4), 456-70. doi: 10.1080/13803395. 2010.533157.

Rey, A. (1958). L'Examen clinique en psychologie. Paris: Press Universitaire de France; 1958.

Roozenbeek, B., Maas, A. I. R., \& Menon, D. K. (2013). Changing patterns in the epidemiology of traumatic brain injury. Nature reviews. Neurology, 9(4), 231-236. doi: 10.1038/nrneurol.2013.22.
Schoenberg, M. R., Dawson, K. A., Duff, K., Patton, D., Scott, J. G., \& Adams, R. L. (2006). Test performance and classification statistics for the Rey Auditory Verbal Learning Test in selected clinical samples. Archives of Clinical Neuropsychology, 21, 693-703. doi: 10.1016/ j.acn.2006. 06.010.

Selassie, A. W., Zaloshnja, E., Langlois, J. A., Miller, T., Jones, P., \& Steiner, C. (2008). Incidence of long-term disability following traumatic brain injury hospitalization. The Journal of Head Trauma Rehabilitation, 23(2), 123-131. doi: 10.1097/01.HTR.0000314531.30401.39.

Skandsen, T., Finnanger, T. G., Andersson, S., Lydersen, S., T, A. S., T., F., \& Andersson, S. (2010). Cognitive impairment 3 months after moderate and severe traumatic brain injury: A prospective follow-up study. Archives of Physical Medicine and Rehabilitation, 91(12), 1904 1913. doi: 10.1016/j.apmr.2010.08.021.

Squire, L., Stark, C. \& Clark, R. (2004). The medial temporal lobe. Annual reviews neuroscience, 27, 279-306. doi: 10.1146/annurev.neuro.27.070203.144130.

Strauss, E., Sherman, E. S., \& Spreen, O. (2006). A compendium of neuropsychological tests - administration, norms, and commentary. New York: Oxford University Press.

Teasdale, G., \& Jennett, B. (1974). Assessment of coma and impaired consciousness - a practical scale. The Lancet, 2(7872), 81-84.

Torres, D. S., Abrantes, J. \& Brandão-Mello, C. E. (2013). Cognitive assessment of patients with minimal hepatic encephalopathy in Brazil. Metabolic brain disease, 28, 473-483. doi: 10.1007/s11011-013-9405-3.

Tulving, E. (2002). Episodic Memory: From mind to brain. Annual Review Psychology, 53(1), 1-25. doi: 10.1146/ annurev.psych.53.100901.135114.

Wood, R. L., \& Liossi, C. (2007). The relationship between general intellectual ability and performance on ecologically valid executive tests in a severe brain injury sample. Journal of the International Neuropsychological Society, 13(1), 90-98. doi: 10.1017/S1355617707070129.

Zappala, G., Schotten, M. T., \& Eslinger, P. J. (2012). Traumatic brain injury and the frontal lobes: What can we gain with diffusion tensor imaging? Cortex, 48(2), 156-165. doi: 10.1016/j.cortex.2011.06.020.

Recebido em: 19/02/2014 Última revisão em: 11/08/2014 Aceito em: 17/08/2014 